\title{
Research on the technology of constructing a protection with joint use of no-fines concrete and bitumen
}

\author{
Andrey Mikhasek ${ }^{1, *}$ \\ ${ }^{1}$ Samara State Technical University, Institute of Architecture and Civil Engineering, \\ 194, Molodogvardeyskaya St., 443001, Samara, Russia.
}

\begin{abstract}
To build a bank slope protection of a hydraulic structure, a technology is proposed that involves building a framework of no-fines concrete and pouring it with hot bituminous materials. Implementation of this technology requires conducting a research on bitumen penetration into the protection plate body. The research involved developing models of non-stationary filtration under varied bitumen viscosity. The research involved experimental observation in laboratory environment. Models of movement of a variable viscosity liquid in a porous medium were proposed, and their study in laboratory environment allowed for identifying the depth of penetration, which is an important result to solve engineering objectives.
\end{abstract}

\section{Introduction}

A search for solutions to the problem of strengthening the waterline to reduce the construction costs is an important and relevant objective [1-3]. A new technology [4] that involves pouring a no-fines concrete plate with hot bitumen is proposed to protect the bank slope of a hydraulic structure.

While moving, the bitumen gets colder and its viscosity changes; this results in velocity reduction and, consequently, the bitumen sticks in the body of no-fines concrete.

Thus, when choosing a model, the following objective (goal) has been set: to identify dependence of the depth of bitumen penetration (in general, a variable viscosity liquid) on its characteristics and the characteristics of no-fines concrete (porous medium).

The depth of viscous liquid penetration into a porous medium will depend on the following parameters:

- the liquid density $\rho$;

- viscosity $\mu$;

- the ratio $\Delta \mathrm{K}$ of the initial liquid temperature to the temperature at the moment of movement termination - the temperature of "sticking";

- effective diameter $\mathrm{d}$ of the medium particles;

- porosity coefficient m;

* Corresponding author: andremixas@mail.ru 
- free fall acceleration $g$.

\section{Materials and Methods}

To reach the first objective, we used a symmetric tube of flow that can be represented as a vertically positioned straight unit tube, centered relative to the axis of symmetry.

Taking into account that the issue of variable viscosity bitumen penetration into nofines concrete has been studied very little, we use the dimension theory method, and then the correlation between the values can be written as follows:

$$
h=f(\mu, \rho, d, g, \Delta K, m),
$$

where $\mathrm{h}$ - depth of variable viscosity liquid penetration into a porous medium, $\Pi$ according to the theorem, any correlation between physical dimensions can be presented as a dependence between $n+1-k$ ( $\mathrm{k}-$ the number of physical values with independent dimensions) and dimensionless values $\Pi$.

Let us take $\mu, \rho, d$ as parameters with independent dimensions. To check their independence, we write down their dimensions and calculate the determiner comprised of the indicators of base dimension exponent values:

$$
[\mu]=L^{-1} M T^{-1}=\left[\frac{\mathrm{kg}}{\mathrm{m} \cdot \mathrm{s}}\right] ;[\rho]=L^{-3} M=\left[\frac{\mathrm{kg}}{\mathrm{m}^{3}}\right] ;[d]=L=[\mathrm{m}] .
$$

The determiner can be represented as follows:

$$
\left|\begin{array}{ccc|c} 
& L & M & T \\
\rho & -3 & 1 & 0 \\
\mu & -1 & 1 & -1 \\
d & 1 & 0 & 0
\end{array}\right| \neq 0
$$

The determiner is other than zero, so the parameters have independent dimensions.

On the basis of the values in brackets, using Buckingham's method, we take $\mathrm{m}, \Delta \mathrm{K}$ for dimensionless values. We obtain dimensionless complex

$$
P=\frac{h}{d}
$$

The dimensionless complex

is obtained.

$$
P_{1}=\frac{g}{\rho^{\alpha} \cdot \mu^{\beta} \cdot d^{\gamma}}
$$

Then we find coefficients $\alpha, \beta, \gamma$ of the dimensionless complex P1.

The numerator and denominator have equal value, so

$$
\frac{g}{\rho^{\alpha} \cdot \mu^{\beta} \cdot d^{\gamma}}=\frac{L \cdot T^{-2}}{L^{-3 \alpha} \cdot M^{\alpha} \cdot L^{-\beta} \cdot M^{\beta} \cdot T^{-\beta} \cdot L^{\gamma}} .
$$

We equate exponent values with equal dimensions and obtain the system of equations: 


$$
\left\{\begin{array}{l}
-3 \alpha-\beta+\gamma=1 \\
\alpha+\beta=0 \\
\beta=2
\end{array} .\right.
$$

We solve this system of equations (5) and obtain:

$$
\left\{\begin{array}{l}
\alpha=-2 \\
\beta=2 \\
\gamma=-3
\end{array} .\right.
$$

We substitute the obtained values into (3) and obtain (5):

$$
P_{1}=\frac{g}{\rho^{-2} \cdot \mu^{2} \cdot d^{-3}}=\frac{\rho^{2} \cdot g \cdot d^{3}}{\mu^{2}} .
$$

The obtained dimensionless complex is a Galileo number [2] that can be otherwise written as:

$$
G a=\frac{\mathrm{Re}^{2}}{F r}=\frac{g \cdot d^{3}}{v^{2}}
$$

or we can express kinematic viscosity in terms of dynamic viscosity

$$
v=\frac{\mu}{\rho}
$$

and obtain:

$$
G a=\frac{g \cdot d^{3} \cdot \rho^{2}}{\mu^{2}}=P_{1}
$$

According to V.V. Gukhman [5], Galileo number describes free flow of liquid, which we believe to most fully reflect the process of viscous liquid penetration into a porous medium due to gravity. Thus, the formula (2) can be represented as:

$$
h=d \cdot f(G a, \Delta K, m) .
$$

The type of dependence (7) can be obtained through processing experimental data that we plan to obtain using models.

\section{Results}

Preparatory work in experiments with no-fines concrete also included concrete preparation directly in the metal tube. To make concrete, we used crushed stone with fraction of 20-40; concrete mix composition and concrete preparation method in laboratory environment are described earlier. The metal tube was greased with oil for further separation of concrete from its surface, and then concrete was laid and compacted, in the same way as crushed stone. The no-fines concrete was cured for 24 hours.

Penetration results were evaluated using a measurement method and presented in Table 1. Then we conducted one-factor dispersion analysis of the obtained results, which is 
presented in Tables 2 and 3.

Table 1. Results of experiments with the bitumen model using no-fines concrete.

\begin{tabular}{|c|c|c|c|}
\hline \multirow{2}{*}{ Experiment No. } & \multicolumn{3}{|c|}{ Penetration depth in $\mathbf{m}$ at the temperature of ${ }^{\circ} \mathbf{C}$} \\
\cline { 2 - 4 } & $\mathbf{1 2 0}$ & $\mathbf{1 6 0}$ & $\mathbf{2 0 0}$ \\
\hline 1 & 0.16 & 0.4 & 0.75 \\
\hline 2 & 0.13 & 0.36 & 0.64 \\
\hline 3 & 0.14 & 0.37 & 0.67 \\
\hline Average value & 0.14 & 0.38 & 0.69 \\
\hline
\end{tabular}

Table 2. Dispersion calculation.

\begin{tabular}{|c|c|c|c|c|}
\hline Groups & Count & Sum & Average & Dispersion \\
\hline Column 1 & 3 & 0.43 & 0.14 & 0.0002 \\
\hline Column 2 & 3 & 1.13 & 0.38 & 0.0004 \\
\hline Column 3 & 3 & 2.06 & 0.69 & 0.003 \\
\hline
\end{tabular}

Table 3. Variation source.

\begin{tabular}{|c|c|c|c|c|c|c|}
\hline Variation source & SS & df & MS & F & P-Value & F critical \\
\hline Between the groups & 0.45 & 2 & 0.223 & 171.4 & $5.08619 \mathrm{E}-06$ & 5.14 \\
\hline In the groups & 0.0078 & 6 & 0.0013 & & & \\
\hline Total & 0.45 & 8 & & & & \\
\hline
\end{tabular}

Thus

$$
\hat{\mathrm{F}}=171.4>\mathrm{F}_{(\mathrm{k}=1 . \mathrm{n}-\mathrm{k}, \alpha)}=5.14,
$$

the penetration depth data with the bitumen model using no-fines concrete and crushed stone with fraction of 20-40 statistically belong to a single functional dependence.

The conducted research on physical models indicates that bitumen movement is affected by the following factors: bitumen density $\rho$, the ratio $\Delta K$ of its initial temperature to the temperature of sticking, viscosity coefficient $\mu$, effective diameter $\mathrm{d}$ of no-fines concrete particles, porosity coefficient $\mathrm{m}$ and free fall acceleration $\mathrm{g}$. Using the dimension theory method, we identified functional dependence of the depth of bitumen penetration into nofines concrete on the parameters indicated in Formula 7. We need to identify the type of this function.

Exponential, logarithmic and direct functions were considered as possible types of functional dependence. Logarithmic function was chosen by trial and error.

Thus, by data fitting, the following type of dependence was identified:

$$
h=d \cdot\left(\ln \frac{G a}{B \cdot \Delta K}\right) \cdot A \cdot m,
$$

where $\mathrm{d}$ - effective diameter of the porous medium particles, $\mathrm{Ga}$ - Galileo number is identified by the dependence, $\Delta K=\frac{K_{i n}}{K_{s t}}$ - the ratio of the temperature at the start of injection Kinitial to the temperature of liquid sticking Ksticking (for water, the ratio is 273 $\mathrm{K}$, for bitumen $-373 \mathrm{~K}), \mathrm{m}$ - porosity coefficient, $\mathrm{A}$ and $\mathrm{B}$ - correction coefficients obtained through the least squares method based on the results of experimental data processing.

\section{Discussion}

On the basis of the conducted research, we obtained the dependence that can be used for 
engineering calculations, namely: [1] process temperature of $170-180{ }^{\circ} \mathrm{C}$ is established and recommended for no-fines concrete used with the aggregate of $80-120 \mathrm{~mm}$, with the layer thickness of $60 \mathrm{~cm}$.

The dependence obtained is valid for different mediums. Yet comparison of penetration depths on different models is possible only with equal values of Ga numbers and equal volumes of the injected liquid.

The proposed method allows to predetermine the layer thickness depending on sealing compounds and penetration depth for no-fines concrete made with different aggregates, though this requires correction of $\mathrm{A}$ and $\mathrm{B}$ coefficients.

The theoretical data obtained were proved by a full-scale experiment on an experimental block of no-fines concrete: bitumen fully penetrated into the whole layer, the penetration depth and the shape of spreading were close to the estimated values.

The results of the research were used to identify the main process parameters [6-10].

\section{Conclusions}

The research conducted showed:

1. The proposed method of building bank slope protection that involves pouring a nofines concrete plate with hot bitumen can be implemented in practice, main process parameters, such as the temperature of bitumen and the size of the aggregate, can be predetermined, and penetration depth can be predicted.

2. The experiment conducted proved that liquid of a certain variable viscosity sticks in porous medium at a certain depth. The experiment conducted on physical models provided us with data on the depth of hot bitumen penetration into no-fines concrete made of crushed stone with fraction of 20-40. It is established that statistical penetration depth depends on temperature. In case of non-stationary processes, the shape of spreading is described as a circle.

\section{References}

1. I.S. Sobol, The Volga Region Scientific Magazine 2 (26), 65-67 (2013)

2. A.V. Mishakova, A.V. Vakhrushkina, V.A. Murgul, T.V. Sazonova, Procedia Engineering 15th. Cep. "15th International Scientific Conference 'Underground Urbanisation as a Prerequisite for Sustainable Development', ACUUS 2016", 18121817 (2016)

3. M.I. Balzannikov, I.S. Kholopov, M.V. Kholopov, M.G. Litvinova, Scientific Survey 22, 65-71 (2015)

4. Andrey Mikhasek, Boris Ivanov, MATEC Web of Conferences 105, 03022 (2017)

5. V.V. Gukhman, Introduction to the Theory of Similitude (Higher School, Moscow, 1963)

6. A.A. Mikhasek, A.A. Smyvalov, Scientific Survey 14, 102-109 (2015)

7. A.A. Mikhasek, M.V. Rodionov, Procedia Engineering 91, 82-88 (2015)

8. A.A. Mikhasek, Scientific Survey 14, 67-72 (2016)

9. A.A. Mikhasek, M.V. Rodionov, V.A. Seliverstov, Y.E. Senitsky, MATEC Web of Conferences 86 (2016)

10. M.I. Balzannikov, M.V. Rodionov, V.A. Seliverstov, Urban Construction and Architecture 1, 100-105 (2011) 